\title{
Isozyme inheritance and variation in Actinidia
}

\author{
HONGWEN HUANG, FENNY DANE* $\dagger$, ZHONGZEN WANG $\ddagger$, ZHENGWAN JIANG, \\ REHUANG HUANG \& SHENGMEI WANG \\ Wuhan Institute of Botany, The Chinese Academy of Sciences, Wuhan, Hubei 430074, China, †Department of \\ Horticulture, Auburn University, Auburn, AL 36849, U.S.A. and $\ddagger$ Institute of Botany, The Chinese Academy of \\ Sciences, Beijing 100093, China
}

\begin{abstract}
Isozyme inheritance and variation in Actinidia was investigated using 23 enzyme systems. Ten isozyme loci from six enzyme systems, $A c p-2$, Est $\operatorname{Prx}-1, \operatorname{Pr} x-2, \operatorname{Prx}-3, \operatorname{Prx}-4, \operatorname{Prx}-5, \operatorname{Pgi}-2, \operatorname{Pgm}-2$ and $T p i$, were found to be inherited as single Mendelian genes in families of two interspecific crosses. Disomic inheritance detected at 10 loci in progenies of a cross between the hexaploid $A$. deliciosa and the diploid $A$. chinensis (Jingkui $\times$ Moshan no. 4) provided convincing evidence that $A$. deliciosa is an allohexaploid. Allelic segregation for tetrasomic inheritance at the 10 isozyme loci was demonstrated in the progenies of a cross between the tetraploid $A$. chinensis and the diploid $A$. eriantha, a result suggesting autoploid origin of the tetraploid $A$. chinensis, which apparently originated from its diploid ancestor $A$. chinensis. A high level of isozyme variation and heterozygosity was observed in the 22 cultivars and 56 plants from 28 Actinidia taxa. Allozyme phenotype can be used effectively for cultivar identification. The application of isozyme markers in the study of phylogeny could still be difficult in polyploids of natural populations in which estimations of gene frequencies are a prerequisite.
\end{abstract}

Keywords: Actinidia, disomic inheritance, isozyme loci, kiwifruit, polyploid, tetrasomic inheritance.

\section{Introduction}

The genus Actinidia contains more than 60 species and 110 taxa (Cui, 1993). The best-known species is A. deliciosa C. F. Liang et A. R. Ferguson, the commercially developed kiwifruit. The natural range of Actinidia is remarkably wide, as it extends from the tropics (latitude $0^{\circ}$ ) to cold temperate regions $\left(50^{\circ} \mathrm{N}\right)$. Actinidia species are found from Siberia to Indonesia, and from India to Japan (Cui, 1993). However, the original distribution of most taxa of Actinidia is centred around the mountains and hills of south-central and south-east China, with the QinLing mountain as the northern boundary and the HengDuan mountain as the western boundary. Fiftynine species, 43 varieties and seven forms have been found in China (Liang, 1983; Ferguson, 1990) Species identification in Actinidia has proven very difficult because morphological differences between species and intraspecific taxa are not always clearcut (Ferguson, 1990). The overlapping geographical distributions and natural hybridization of taxa have

*Correspondence. E-mail: fdane@ag.auburn.edu also produced various transitional forms between varieties of the same species.

All species of Actinidia are dioecious but show variations in ploidy level. Diploid $(2 n=58)$, tetraploid $(2 n=4 \mathrm{x}=116)$ and hexaploid $(2 n=6 \mathrm{x}=174)$ species are known. In several species, there is evidence for intrataxon variation in ploidy level (Xiong et al., 1985; Yan et al., 1994). Dioecism and ploidy level variation greatly hinder the genetic study of heritable traits, particularly flower and fruit traits, which are usually very useful for the identification of cultivars and species. Although $A$. deliciosa $(6 \mathrm{x})$ is well known throughout the world as a successful commercial crop, inheritance of most of its important traits remains unknown so far. Also, the mode of inheritance of the commercially important species, such as $A$. deliciosa $(6 \mathrm{x})$ and $A$. chinensis $(2 \mathrm{x}, 4 \mathrm{x})$, remains unclear. The majority of the breeding programmes for cultivar improvement depend largely on extensive selections from natural germplasm populations. A national germplasm repository of Actinidia and breeding programme for kiwifruit cultivar improvement were initiated in 1978 at the Wuhan Institute of Botany with, as goals, the 
preservation of natural resources of Actinidia in China and the development of superior cultivars with higher quality, stress tolerance and pest resistance. It is, therefore, important to develop simply inherited markers, such as isozymes, for studying genetics, because a knowledge of chromosome behaviour and mode of inheritance at different ploidy levels is a prerequisite for breeding strategies and will also provide information about phylogenetic relationships within the genus Actinidia.

Isozymes offer several applications in studies of both intraspecific and interspecific phylogenetic systematics (Murphy et al., 1990). Isozymes have also been used extensively in other fruit tree breeding programmes as genetic markers for the identification of cultivars (Degani et al., 1995), the confirmation of hybridity (Anderson et al., 1991), marker-assisted selection (Manganaris et al., 1994) and many other aspects of plant breeding programmes (Tanksley \& Orton, 1983). However, no attempt has been made to study isozymic inheritance and variations in Actinidia. The only published work to date reported the use of aspartate aminotransferase (AAT), phosphoglucomutase (PGM) and phosphoglucoisomerase (PGI) for the identification of nine kiwifruit cultivars (Messina et al., 1991). The purpose of the present study was (i) to establish isozyme markers for Actinidia species by progeny testing of controlled crosses and (ii) to evaluate isozyme variation in a wide array of accessions of Actinidia in our repository.

\section{Materials and methods}

\section{Plant material}

Progenies derived from two interspecific crosses were used for genetic analysis. The JM family (106 progenies) was developed from the controlled cross between the pistillate cultivar Jingkui (6x) of $A$. deliciosa and the staminate cultivar Moshan no. 4 (2x) of $A$. chinensis Planch. These two species are closely related and completely cross-compatible. The WE family (60 progenies) was developed by crossing a staminate plant of $A$. eriantha Benth. (2x) with the pistillate cultivar Wuzhi no. 3 of $A$. chinensis $(4 \mathrm{x})$. The hybridization techniques for interspecific crosses in Actinidia have been described previously by Wang et al. (1994). Twenty-eight taxa representing three sections of the genus (Table 4) were included in this study for the evaluation of isozyme variability. Actinidia deliciosa and $A$. chinensis, however, were represented by 10 and 12 cultivars respectively (Table 3 ). Typically, one male and one female plant of each taxon was used for analysis. Plants of all taxa and the progenies were at least 2 years old. The plants were grown at a $3 \times 4 \mathrm{~m}$ spacing in experimental field plots at the Wuhan Institute of Botany, Wuhan, China.

\section{Electrophoresis and isozyme staining}

Isozyme assays were conducted on winter dormant buds. The extraction procedure, as described by Huang et al. (1994), and the isoelectric focusing polyacrylamide slab gel system of $\mathrm{pH} 4-9$, as described by Mulcahy et al. (1981), were used in this study because of their high resolution and efficiency (100 samples per run), with the exception of phosphoglucoisomerase (PGI) and phosphoglucomutase (PGM) assays, which were conducted on 12 per cent starch gels with a pH 6.1 morpholine-citrate buffer (Wendel \& Weeden, 1989). Gels were assayed for acid phosphatase (ACP; EC 3.1.3.2), alcohol dehydrogenase (ADH; EC 1.1.1.1), aconitate hydratase (ACO; EC 4.2.1.3), adenylate kinase (ADK; EC 2.7.4.3), esterase (EST; EC 3.1.1.-), formate dehydrogenase (FDH; EC 1.2.1.2), fructose-bisphosphate aldolase (FBA; EC 4.1.2.13), fructose bisphosphatase (FBP; EC 3.1.3.11), fumarate hydratase (FUM; EC 4.2.1.2), glucose-6-phosphate dehydrogenase (G6PDH; EC 1.1.1.49), glutamate dehydrogenase (GDH; EC 1.4.1.2), glyceraldehyde-3phosphate dehydrogenase (G3PDH; EC 1.2.1.12), hexokinase (HEX; EC 2.7.1.1), isocitrate dehydrogenase (IDH; EC 1.1.1.41), malate dehydrogenase (MDH; EC 1.1.1.37), malic enzyme (ME; EC 1.1.1.40), mannose-6-phosphate isomerase (MPI; EC 5.3.1.8), peroxidase (PRX; EC 1.11.1.7), phosphoglucomutase (PGM; EC 5.4.2.2), phosphogluconate dehydrogenase (PGD; EC 1.1.1.44), phosphoglucoisomerase (PGI; EC 5.3.1.9), shikimate dehydrogenase (SKD; EC 1.1.1.25) and triose-phosphate isomerase (TPI; EC 5.3.1.1). Staining recipes were those of Wendel \& Weeden (1989), with minor pH and ingredient concentration modifications.

\section{Allozyme designation and data analysis}

The genetic control of isozyme loci was postulated based on the phenotypic banding patterns and the segregation ratios of the controlled crosses. The loci for a given multilocus enzyme system were designated sequentially by number starting with 1 for the most anodal locus; at each locus, the alleles were designated sequentially by letter starting with $a$ for the most anodal allozyme. Allozymic phenotypes were designated by capital letters corresponding to 
the same alleles (such as phenotype $\mathrm{AB}$ corresponding to $a b, a a a b, a a b b$ or $a b b b$ genotypes). Irregularly spaced rare bands found occasionally in some taxa were designated as rare allele ( $R$ ) by number starting with 1 for the most anodal allele (such as R1). Chi-square tests were used to determine the goodness-of-fit of segregating loci to expected Mendelian segregation ratios. In case of a two-class segregation ratio, chi-squares were calculated using Yates' correction.

\section{Results and discussion}

\section{Inheritance and variation}

Of the 23 enzymes assayed in this study, seven (ACP, EST, MDH, PRX, PGI, PGM and TPI) produced high-resolution banding patterns. The following 16 enzymes were either poorly resolved or of weak intensity and were excluded from further discussion: $\mathrm{ADH}, \mathrm{ACO}, \mathrm{ADK}, \mathrm{FDH}, \mathrm{FBA}, \mathrm{FBP}$, FUM, G3PDH, G6PDH, GDH, HEX, IDH, ME, MPI, PGD and SKD. Polymorphism was observed at all of the seven well-resolved enzyme systems. Genetic inferences made to explain the variability at these enzyme systems were based on the banding patterns observed in the segregating progenies. However, for $\mathrm{MDH}$, segregations were not observed in the crosses, and the complex zymogram patterns of the cultivars and species provided no inference about its genetics. ACP, EST, PGM and PRX were found to be monomeric enzymes, whereas PGI and TPI were dimeric enzymes in Actinidia. The inheritance at each locus and the variation detected among

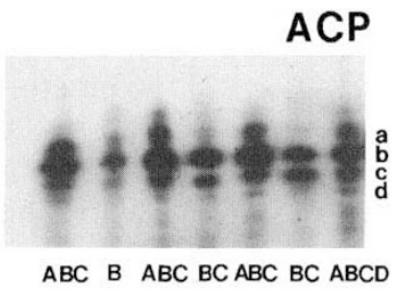

EST
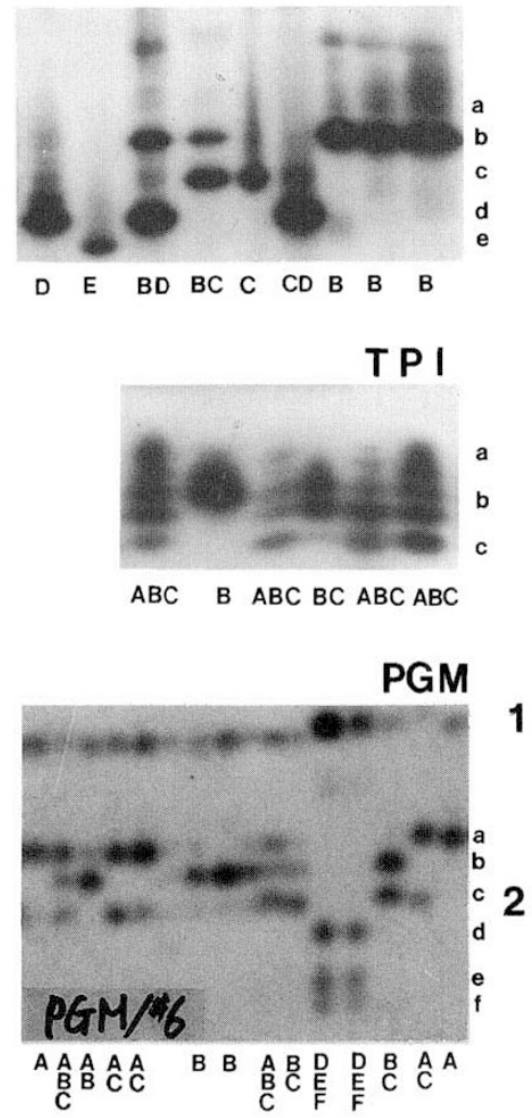

PG I

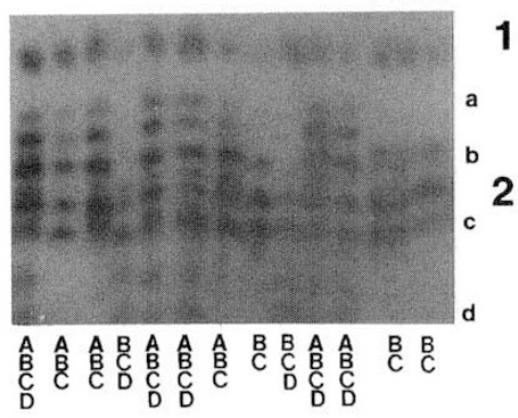

PRX

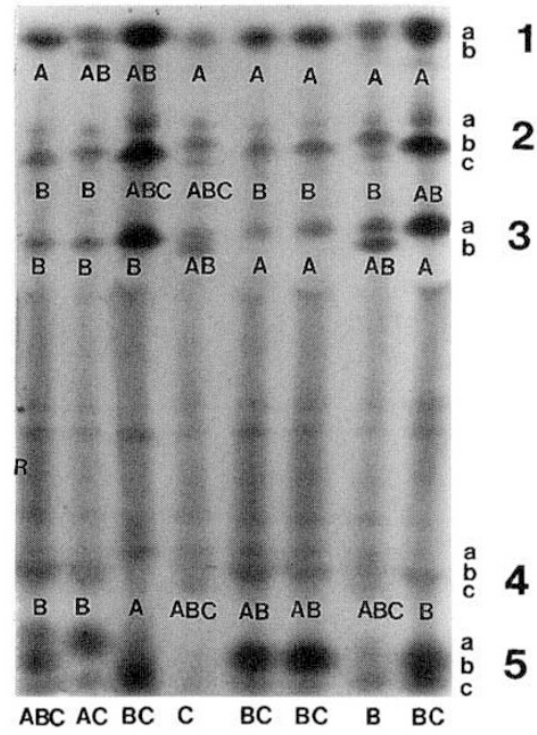

Fig. 1 Allele and locus designations and allozyme phenotypes of 12 isozyme loci in Actinidia. 
cultivars and different species will be discussed below.

\section{Esterase (EST)}

In the $\mathrm{JM}$ family $[A$. deliciosa $(6 \mathrm{x}) \times A$. chinensis $(2 \mathrm{x})$ ], progenies segregated for either single- or double-banded phenotypes and gave the expected Mendelian segregation ratio of 1:2:1 (Fig. 1 and Table 1). This result is consistent with the disomic mode of inheritance of an allohexaploid species. Analysis of one repeat DNA sequence in $A$. deliciosa recently suggested the alloploid nature of this species (Crowhurst \& Gardner, 1991). In the WE family $[A$. chinensis $(4 \mathrm{x}) \times A$. eriantha $(2 \mathrm{x})]$, three banding patterns, single-, double- and triple-banded, were observed in the progenies. Segregation data for this family fit the expected phenotypic ratios for a tetrasomic mode of inheritance of three alleles at this locus (Table 2). Both diploid and tetraploid plants have been found in $A$. chinensis (Yan et al., 1994). The tetrasomic inheritance of the Est allozymes points to the autoploid origin of the tetraploid $A$. chinensis from a diploid $A$. chinensis.

Table 1 Segregation and $\chi^{2}$ goodness-of-fit test for disomic inheritance at 10 isozyme loci in the JM family from the interspecific cross Actinidia deliciosa $\times$ A. chinensis

\begin{tabular}{|c|c|c|c|c|c|c|}
\hline \multirow[b]{2}{*}{ Locus } & \multicolumn{2}{|c|}{ Parental } & \multirow[b]{2}{*}{ Expected gamete ratios } & \multirow[b]{2}{*}{ Expected phenotypic ratios } & \multirow[b]{2}{*}{ Progeny phenotypes } & \multirow[b]{2}{*}{$\chi^{2}$} \\
\hline & Phenotype & Genotype & & & & \\
\hline Acp-2 & $\mathrm{BC} \times \mathrm{B}$ & $---b c \dagger \times b b$ & $(1--b: 1-c) \times(b)$ & 1B:1BC & 58B:43BC:5ABC? $\ddagger$ & $2.481 \mathrm{NS}$ \\
\hline Est & $\mathrm{BC} \times \mathrm{BC}$ & $---b c \times b c$ & $(1--b: 1--c) \times(1 b: 1 c)$ & 1B:2BC:1C & 22B:50BC:34C & 3.057 NS \\
\hline$P g i-2$ & $\mathrm{ABC} \times \mathrm{BC}$ & $a b c-b c \times b c$ & $(1 a b c: 1-b c) \times(1 b: 1 c)$ & $1 \mathrm{ABC}: 1 \mathrm{BC}$ & 58ABC:48BC & $0.943 \mathrm{NS}$ \\
\hline$P g m-2$ & $\mathrm{BCD} \times \mathrm{BD}$ & $-c d-c d \times b d$ & $(1-c b: 1-c d) \times(1 b: 1 d)$ & 1BC:2BCD:1CD & 17BC:63BCD:26CD & $5.303 \mathrm{NS}$ \\
\hline $\operatorname{Pr} x-1$ & $\mathrm{AB} \times \mathrm{AB}$ & $--a b \times a b$ & $(1--a: 1--b) \times(1 a: 1 b)$ & 1A:2AB:1B & 25A:55AB:26B & $0.169 \mathrm{NS}$ \\
\hline $\operatorname{Prx}-2$ & $A \times B$ & $---a \times b b$ & $(--a) \times(b)$ & $\mathrm{AB}$ & $106 \mathrm{AB}$ & \\
\hline $\operatorname{Pr} x-3$ & $\mathrm{AB} \times \mathrm{A}$ & $---a b \times a a$ & $(1-a: 1-b) \times(a)$ & $1 \mathrm{~A}: 1 \mathrm{AB}$ & 51A:55AB & $0.151 \mathrm{NS}$ \\
\hline$P r x-4$ & $\mathrm{BC} \times \mathrm{C}$ & $---b c \times c c$ & $(1--b: 1--c) \times(c)$ & $1 \mathrm{BC}: 1 \mathrm{C}$ & 55BC:51C & $0.151 \mathrm{NS}$ \\
\hline $\operatorname{Prx}-5$ & $\mathrm{~B} \times \mathrm{C}$ & $-\cdots b \times c c$ & $(--b) \times(c)$ & $\mathrm{BC}$ & $106 \mathrm{BC}$ & \\
\hline$T p i$ & $\mathrm{BC} \times \mathrm{BC}$ & $---b c \times b c$ & $(1--b: 1-c) \times(1 b: 1 c)$ & 1B:2BC:1C & 28B:49BC:29CC & $0.623 \mathrm{NS}$ \\
\hline
\end{tabular}

$\dagger$ Indicates a null or monomorphically duplicated allele.

¥Unexpected phenotype class.

Table 2 Segregation and $\chi^{2}$ goodness-of-fit test for tetrasomic inheritance at 10 isozyme loci in the WE family from the interspecific cross Actinidia chinensis $\times$ A. eriantha

Parental

Locus Phenotype Genotype Expected gamete ratios Expected phenotypic ratios Progeny phenotypes $\quad \chi^{2}$

\begin{tabular}{|c|c|c|c|c|c|c|}
\hline$A c p-2$ & & & & 3D:4BCD:1CD & $\mathrm{BCD}: 6 \mathrm{CD}: 6 \mathrm{BC} ? \dagger$ & $7.125^{*}$ \\
\hline Est & $\mathrm{BC} \times \mathrm{AC}$ & & & $\mathrm{ABC}: 1 \mathrm{BC}: 1 \mathrm{CC}$ & $4 \mathrm{ABC}: 16 \mathrm{BC}: 22 \mathrm{C}$ & \\
\hline Pgi-2 & $\mathrm{CDE} \times \mathrm{CE}$ & & $\times(1 c: 1 e)$ & CDE:1DE & 8C:8CD:25CE:17CDE:0DE & \\
\hline$m-2$ & $\mathrm{BD} \times \mathrm{B}$ & & & & D:3BCD?:3BC? & NS \\
\hline $\operatorname{Prx}-1$ & $\mathrm{AB} \times$ & $a a a b$ & & & & \\
\hline $\operatorname{Pr} x-2$ & $\mathrm{ABC} \times \mathrm{BC}$ & & & BC:3ABC:1B & $B: 1$ & 6.6 \\
\hline$P r x-3$ & & & & & & \\
\hline & & & & & & \\
\hline Prx -5 & & & $(1 a b: 2 a c: 2 b c: 1 c c) \times(1 b: 1 c)$ & BC:2AC:1C & & \\
\hline$T p i$ & $\mathrm{ABC} \times \mathrm{A}$ & $a b c c \times a a$ & $(1 a b: 2 a c: 2 b c: 1 c c) \times(a)$ & $1 \mathrm{AB}: 3 \mathrm{AC}: 2 \mathrm{ABC}$ & 11AB:32AC:17ABC & $1.883 \mathrm{NS}$ \\
\hline
\end{tabular}

†Unexpected phenotype class.

*Significant at $P<0.05$.

(C) The Genetical Society of Great Britain, Heredity, 78, 328-336. 
Theoretically, autotetraploids can carry up to four different alleles per locus. The triallelic genotype expressed as a triple-banded phenotype simply indicates the monomeric nature of the Est locus. The genetic designation of the $a, b$ and $c$ alleles was based on results from the two crosses. Of the 22 cultivars examined, 55 per cent of the cultivars were heterozygous for the $b$ and $c$ alleles and showed the BC phenotype (bbcc, bbbc, bccc) (Table 3). Allozymic genotyping of these heterozygotes requires additional information from cytogenetic studies and allelic segregation analysis from controlled crosses. Of the 28 taxa in which one male and one female plant were examined, the $a$ allele was found in three taxa only: $A$. callosa var. discolor, $A$. zhejiangensis and $A$. grandiflora. Two additional cathodal bands, putatively designated alleles $d$ and $e$ (cathodal to $c$ ), were observed in five taxa $(A$. cylindrica var. reticulata, $A$. fulvicoma, $A$. callosa var. discolor, $A$. melanandra and $A$. arguta) and three taxa ( $A$. polygama, $A$. callosa var. discolor and $A$. valvata), respectively (Fig. 1 and Table 4). The majority of plants were heterozygotes and had the $b$ allele in common, whereas 32 per cent and 9 per cent of plants were found to be homozygous for the $c$ and $b$ alleles, respectively. This Est locus appears to be homologous in all Actinidia species.

\section{Acid phosphatase (ACP)}

One polymorphic zone, consisting of either singleor double-banded phenotypes, was observed in the progenies of JM (A. deliciosa $\times A$. chinensis), and an additional triple-banded phenotype was observed in the WE $(A$. chinensis $\times A$. eriantha $)$ family. The expected phenotypic ratio of the JM family followed a disomic inheritance pattern (Table 1), whereas segregation data for the WE family, in which the expected phenotypic ratio was tested for tetrasomic inheritance, were skewed with marginal significance at the 5 per cent level (Table 2). This can be attributed to preferential segregation in an interspecific cross between less related species, as 10 per cent of an unexpected maternal phenotype (BC) was found in the progenies. A more anodal band was also detected among the cultivars and species. Four alleles can be tentatively designated $a, b, c$ and $d$ for this locus. Considerable variation in banding pattern was observed among the 22 cultivars and 28 taxa examined (Tables 3 and 4). In addition to the zone

Table 3 Isozyme phenotypes of 22 Actinidia cultivars

\begin{tabular}{|c|c|c|c|c|c|c|c|c|c|c|c|}
\hline Cultivar & Species $\dagger$ & ACP-2 & EST & PGI-2 & PGM-2 & PRX-1 & PRX-2 & PRX-3 & PRX-4 & PRX-5 & TPI \\
\hline Sanxia no. 1 & de & $\mathrm{BC}$ & C & $\mathrm{AC}$ & $\mathrm{BCDE}$ & A & BC & $\mathrm{AB}$ & $\mathrm{BC}$ & A & $\mathrm{BC}$ \\
\hline Wuzhi no. 1 & ch & $\mathrm{BC}$ & $\mathrm{C}$ & $\mathrm{ABD}$ & $\mathrm{BCD}$ & $\mathrm{AB}$ & $\mathrm{AB}$ & $\mathrm{AB}$ & A & $\mathrm{ABC}$ & $\mathrm{ABC}$ \\
\hline Daguohongxin & de & $\mathrm{AB}$ & $\mathrm{C}$ & $\mathrm{BD}$ & $\mathrm{BCD}$ & $\mathrm{AB}$ & B & A & $\mathrm{BC}$ & $\mathrm{AC}$ & $\mathrm{ABC}$ \\
\hline Jianmmia no. 1 & de & $\mathrm{BC}$ & $\mathrm{BC}$ & $\mathrm{BC}$ & $\mathrm{BCD}$ & A & B & A & $\mathrm{C}$ & $\mathrm{C}$ & $\mathrm{BC}$ \\
\hline $79-1$ & $\mathrm{ch}$ & B & C & $\mathrm{ABCE}$ & BCD & A & $\mathrm{AB}$ & A & $\mathrm{AB}$ & C & $\mathrm{BCD}$ \\
\hline Tongshan no. 5 & ch & B & $\mathrm{C}$ & $\mathrm{ABCD}$ & $\mathrm{DE}$ & A & $\mathrm{AB}$ & A & B & $\mathrm{AC}$ & BC \\
\hline Moshan no. 7 & ch & $\mathrm{BC}$ & $\mathrm{BC}$ & $\mathrm{BCDE}$ & BC & A & A & A & B & $\mathrm{AC}$ & $\mathrm{BC}$ \\
\hline Xuxiang & de & $\mathrm{BC}$ & $\mathrm{C}$ & $\mathrm{ACE}$ & $\mathrm{BCD}$ & $\mathrm{AB}$ & $\mathrm{AB}$ & A & $\mathrm{BC}$ & $\mathrm{ABC}$ & $\mathrm{BC}$ \\
\hline Wuzhi no. 5 & ch & $\mathrm{BC}$ & $\mathrm{BC}$ & $\mathrm{ABCD}$ & $\mathrm{BD}$ & $\mathrm{AB}$ & B & A & B & $\mathrm{AC}$ & $\mathrm{ABCD}$ \\
\hline Wuzhi no. 3 & ch & $\mathrm{BC}$ & $\mathrm{BC}$ & $\mathrm{CDE}$ & $\mathrm{BD}$ & $\mathrm{AB}$ & $\mathrm{ABC}$ & A & $\mathrm{ABC}$ & $\mathrm{ABC}$ & $\mathrm{ABC}$ \\
\hline Jianhong no. 1 & de & B & B & BCDE & B & A & $\mathrm{AB}$ & A & $\mathrm{C}$ & $\mathrm{C}$ & $\mathrm{BC}$ \\
\hline Sanxia (male) & de & $\mathrm{BC}$ & $\mathrm{BC}$ & $\mathrm{AB}$ & $\mathrm{BCD}$ & $\mathrm{AB}$ & $\mathrm{AB}$ & A & $\mathrm{BC}$ & $\mathrm{AC}$ & $\mathrm{BCD}$ \\
\hline Miliang & de & $\mathrm{BC}$ & $\mathrm{BC}$ & $\mathrm{AB}$ & $\mathrm{BCD}$ & $\mathrm{AB}$ & B & $\mathrm{AB}$ & $\mathrm{BC}$ & $\mathrm{AC}$ & $\mathrm{BD}$ \\
\hline Guihai no. 4 & ch & $\mathrm{AB}$ & $\mathrm{C}$ & $\mathrm{BC}$ & $\mathrm{BC}$ & A & $\mathrm{AB}$ & A & $\mathrm{C}$ & $\mathrm{AC}$ & $\mathrm{AC}$ \\
\hline Chengyang no. 4 & ch & B & $\mathrm{BC}$ & $\mathrm{AC}$ & BD & $\mathrm{AB}$ & B & A & $\mathrm{BC}$ & $\mathrm{C}$ & $\mathrm{AC}$ \\
\hline Qinmei & de & ABC & $\mathrm{BC}$ & $\mathrm{BCDE}$ & $\mathrm{BCD}$ & $\mathrm{ABC}$ & $\mathrm{ABC}$ & $\mathrm{AB}$ & $\mathrm{C}$ & $\mathrm{C}$ & $\mathrm{ABC}$ \\
\hline Wuzhi no. 6 & ch & $\mathrm{BCD}$ & $\mathrm{BC}$ & $\mathrm{CD}$ & $\mathrm{BCD}$ & $\mathrm{AB}$ & B & A & $\mathrm{BC}$ & $\mathrm{AC}$ & $\mathrm{ABCD}$ \\
\hline Qingyuanqiucui & ch & $? ¥$ & $?$ & $\mathrm{AD}$ & $\mathrm{BC}$ & A & $\mathrm{AB}$ & A & $\mathrm{C}$ & A & $?$ \\
\hline Wuzhi no. 2 & ch & B & $\mathrm{BC}$ & $\mathrm{AB}$ & $\mathrm{BCD}$ & A & $\mathrm{AB}$ & A & $\mathrm{C}$ & $\mathrm{ABC}$ & $\mathrm{BCD}$ \\
\hline Changan no. 4 & de & B & B & $\mathrm{AC}$ & $\mathrm{BCDE}$ & B & B & A & $\mathrm{C}$ & $\mathrm{ABC}$ & $\mathrm{BC}$ \\
\hline Hayward & de & $\mathrm{BC}$ & $\mathrm{BC}$ & BCD & BCDE & $\mathrm{AB}$ & B & A & $\mathrm{C}$ & $\mathrm{BC}$ & $\mathrm{BC}$ \\
\hline $81-24$ & ch & B & $\mathrm{BC}$ & $\mathrm{ABE}$ & $\mathrm{BCD}$ & $\mathrm{AB}$ & B & A & BC & $\mathrm{ABC}$ & $\mathrm{BC}$ \\
\hline
\end{tabular}

†de, A. deliciosa; ch, A. chinensis.

¥Undecided phenotype. 
Table 4 Isozyme phenotypes of 28 taxa of Actinidia

\begin{tabular}{|c|c|c|c|c|c|c|c|c|c|c|c|}
\hline \multirow{2}{*}{$\begin{array}{l}\text { Taxa } \\
\text { Sect. Leiocarpae }\end{array}$} & \multicolumn{2}{|c|}{ ACP-2 EST } & \multirow[t]{2}{*}{ PGI-2 } & \multicolumn{3}{|c|}{ PGM-2 PRX-1 PRX-2 } & 2 PRX-3 & PRX-4 & \multicolumn{2}{|c|}{ PRX-5 TPI } & \multirow{3}{*}{$\begin{array}{l}\text { Polyploidy } \dagger \\
\text { cyto. } 2 x, 4 x, 6 x \\
\text { cyto. } 4 x\end{array}$} \\
\hline & BCD & CD & & $\mathrm{AB}$ & $\mathrm{AB}$ & B & & & & & \\
\hline $\begin{array}{l}\text { A. arguta var. arguta } \\
\text { A. macrosperma var. macrosperma }\end{array}$ & $\mathrm{ABC}$ & $\mathrm{BC}$ & ABD & EFG & $A$ & $\mathrm{~B}$ & $\begin{array}{l}\mathrm{D} \\
\mathrm{C} \\
\mathrm{BCD}\end{array}$ & $\stackrel{-}{\mathrm{B}}$ & $\mathrm{C}$ & $\mathrm{ABC}$ & \\
\hline A. macrosperma var. mumoides & $\mathrm{AB}$ ? & $\mathrm{C}$ & BCD & AEFG & $\mathrm{AB}$ & B & $\mathrm{AB}$ & C & $\mathrm{C}$ & A & poly- \\
\hline A. melanandrc & $\mathrm{ABC}$ & $\begin{array}{l}\text { CD } \\
\mathrm{D}\end{array}$ & $\mathrm{ABCD}$ & $\mathrm{AR} 4 \$ \mathrm{C}$ & & $\stackrel{\mathrm{B}}{\mathrm{ABC}}$ & $\begin{array}{l}\mathrm{BCD} \\
\mathrm{AB}\end{array}$ & $\begin{array}{l}\mathrm{C} \\
\mathrm{CD}\end{array}$ & $\begin{array}{l}\mathrm{C} \\
\mathrm{AC}\end{array}$ & $\begin{array}{l}\mathrm{B} \\
\mathrm{ABC}\end{array}$ & cyto. $4 x$ \\
\hline $\begin{array}{l}\text { A. polygama } \\
\text { A. valvata var. valvata }\end{array}$ & $\begin{array}{l}\mathrm{A} \\
\mathrm{AB}\end{array}$ & $\stackrel{\mathrm{E}}{\mathrm{CE}}$ & $\begin{array}{l}\mathrm{BCD} \\
\mathrm{BC}\end{array}$ & $\begin{array}{l}\mathrm{B} \\
\mathrm{BCEF}\end{array}$ & $\begin{array}{l}\mathrm{AB} \\
\mathrm{AB}\end{array}$ & $\begin{array}{l}\text { B } \\
\text { ABCD } \\
\text { ACD }\end{array}$ & $\begin{array}{l}\mathrm{B} \\
\mathrm{ABCD}\end{array}$ & $\begin{array}{l}\mathrm{B} \\
\mathrm{BCD}\end{array}$ & $\begin{array}{l}\mathrm{AC} \\
\mathrm{C} \\
\mathrm{BC}\end{array}$ & $\begin{array}{l}\mathrm{ABC} \\
\mathrm{ABC}\end{array}$ & $\begin{array}{l}\text { cyto. } 2 \mathrm{x}, 4 \mathrm{x} \\
\text { cyto. } 4 \mathrm{x}\end{array}$ \\
\hline $\begin{array}{l}\text { Sect. Maculatae } \\
\text { A. callosa var. discolor }\end{array}$ & $\begin{array}{l}\mathrm{C} \\
\mathrm{BC}\end{array}$ & $\stackrel{\mathrm{AB}}{\mathrm{BCDE}}$ & $\begin{array}{l}\mathrm{CE} \\
\mathrm{BD}\end{array}$ & $\begin{array}{l}\text { R1R2A } \\
\text { AC }\end{array}$ & $\mathrm{A}$ & $\underset{B}{A C}$ & $\mathrm{AB}$ & $\begin{array}{l}\mathrm{BC} \\
\mathrm{ABC}\end{array}$ & $\begin{array}{l}\mathrm{BC} \\
\mathrm{ABD}\end{array}$ & $\mathrm{BC}$ & cyto. $4 \mathrm{x}$ \\
\hline A. callosa var. henryi & $\mathrm{ABC}$ & B & $\mathrm{RCD}$ & $\mathrm{AB}$ & A & $\mathrm{AB}$ & B & $\mathrm{B}$ & $\mathrm{C}$ & B & cyto. $2 x, 4 x$ \\
\hline A. chrysantha & $\begin{array}{l}\mathrm{ABC} \\
\mathrm{ABCD}\end{array}$ & ${ }_{\mathrm{C}}^{\mathrm{BC}}$ & $\mathrm{DE}$ & $\mathrm{R} 4 \mathrm{C}$ & $\begin{array}{l}\mathrm{A} \\
\mathrm{AB}\end{array}$ & $\begin{array}{l}\mathrm{B} \\
\mathrm{BC}\end{array}$ & $\mathrm{BC}$ & $\stackrel{\text { B }}{\mathrm{C}}$ & $\begin{array}{l}\mathrm{AC} \\
\mathrm{BC}\end{array}$ & $\begin{array}{l}\mathrm{AB} \\
\mathrm{BC}\end{array}$ & cyto. $4 \mathrm{x}$ \\
\hline $\begin{array}{l}\text { A. cylindrica var. reticulata } \\
\text { A fasciculoides var fasciculoides }\end{array}$ & $\mathrm{BC}$ & BD & $\mathrm{CD}$ & $\underset{\mathrm{B}}{\mathrm{B}}$ & $\mathrm{AB}$ & B & $\mathrm{AB}$ & $\stackrel{\mathrm{B}}{\mathrm{C}}$ & $\mathrm{B}$ & $\mathrm{ABC}$ & poly- \\
\hline $\begin{array}{l}\text { A. rasciculotaes var. Jasciculotaes } \\
\text { A. nfa }\end{array}$ & $\mathrm{ABC}$ & $\begin{array}{l}\mathrm{B} \\
\mathrm{BC}\end{array}$ & $\begin{array}{l}\mathrm{BCDE} \\
\mathrm{CDE}\end{array}$ & $\mathrm{B}$ & $\stackrel{A}{A}$ & $\stackrel{?}{\mathrm{~A} B}$ & $\stackrel{\text { B }}{\mathrm{BC}}$ & $\begin{array}{l}\mathrm{C} \\
\mathrm{C} \\
\mathrm{BC}\end{array}$ & $\begin{array}{l}\mathrm{BC} \\
\mathrm{B} \\
\mathrm{BC}\end{array}$ & $\begin{array}{l}\mathrm{BC} \\
\mathrm{ABC}\end{array}$ & poly- \\
\hline A. rubricaulis var. carica & $\begin{array}{l}\mathrm{AB} \\
\mathrm{BC}\end{array}$ & $\mathrm{BC}$ & $\mathrm{BD}$ & $\mathrm{R} 3 \mathrm{~A}$ & $\mathrm{AB}$ & $\mathrm{AB}$ & $A B$ & $\begin{array}{l}\mathrm{B} \\
\mathrm{BC}\end{array}$ & $\mathrm{BC}$ & $\begin{array}{l}\mathrm{A} \\
\mathrm{BC}\end{array}$ & \\
\hline A. sabiae folia & $\mathrm{BC}$ & $\mathrm{BC}$ & $\mathrm{AB}$ & $\mathrm{CD}$ & A & B & $\mathrm{BC}$ & $\mathrm{C}$ & $\mathrm{BC}$ & $\mathrm{BC}$ & cyto. $2 \mathrm{x}$ \\
\hline $\begin{array}{l}\text { Sect. Stellatae } \\
\text { A. chinensis var. chinensis }\end{array}$ & $\begin{array}{l}\mathrm{B} \\
\mathrm{AB} \\
\mathrm{BC} \\
\mathrm{C} \\
\mathrm{BCD}\end{array}$ & $\begin{array}{l}\mathrm{BC} \\
\mathrm{C}\end{array}$ & $\begin{array}{l}\text { ABCE } \\
\text { ABD } \\
\text { AB } \\
\text { ABCD } \\
\text { BCDE } \\
\text { CDE } \\
\text { AC } \\
\text { CD } \\
\text { AD } \\
\text { ABE }\end{array}$ & $\begin{array}{l}\text { BCD } \\
\text { DE } \\
\text { BC } \\
\text { BD } \\
\end{array}$ & $\begin{array}{l}\mathrm{A} \\
\mathrm{AB}\end{array}$ & $\begin{array}{l}\mathrm{A} \\
\mathrm{AB} \\
\mathrm{B} \\
\mathrm{ABC}\end{array}$ & A & $\begin{array}{l}\mathrm{A} \\
\mathrm{AB} \\
\mathrm{B} \\
\mathrm{BC} \\
\mathrm{ABC} \\
\mathrm{C}\end{array}$ & $\begin{array}{l}A \\
A C \\
A B C \\
C\end{array}$ & $\begin{array}{l}A C \\
A B C \\
B C \\
B C D \\
A B C D\end{array}$ & cyto. $2 x, 4 x$ \\
\hline A. deliciosa var. deliciosa & $\begin{array}{l}\mathrm{AB} \\
\mathrm{B} \\
\mathrm{ABC} \\
\mathrm{BC} \\
\mathrm{C}\end{array}$ & $\begin{array}{l}\mathrm{BC} \\
\mathrm{C}\end{array}$ & $\begin{array}{l}\mathrm{AC} \\
\mathrm{BD} \\
\mathrm{BC} \\
\mathrm{ACE} \\
\mathrm{BCDE} \\
\mathrm{AB} \\
\mathrm{BCD}\end{array}$ & $\begin{array}{l}\text { BCDE } \\
\text { BCD } \\
\text { B }\end{array}$ & $\begin{array}{l}\mathrm{A} \\
\mathrm{AB} \\
\mathrm{ABC} \\
\mathrm{B}\end{array}$ & $\begin{array}{l}\mathrm{AB} \\
\mathrm{B} \\
\mathrm{BC} \\
\mathrm{ABC}\end{array}$ & $\begin{array}{l}\mathrm{A} \\
\mathrm{AB}\end{array}$ & $\begin{array}{l}\mathrm{BC} \\
\mathrm{C}\end{array}$ & $\begin{array}{l}\mathrm{A} \\
\mathrm{AC} \\
\mathrm{ABC} \\
\mathrm{C} \\
\mathrm{BC}\end{array}$ & $\begin{array}{l}\mathrm{BC} \\
\mathrm{ABC} \\
\mathrm{BCD} \\
\mathrm{BD}\end{array}$ & cyto. $6 \mathrm{x}$ \\
\hline $\begin{array}{l}\text { A. deliciosa var. chlorocarpa } \\
\text { A. eriantha var. eriantha }\end{array}$ & $\stackrel{\mathrm{BC}}{\mathrm{C}}$ & $\begin{array}{l}\mathrm{C} \\
\mathrm{BC} \\
\mathrm{C}\end{array}$ & $\begin{array}{l}\mathrm{ABCD} \\
\mathrm{CD}\end{array}$ & ${ }_{B}^{A B C}$ & $\begin{array}{l}\mathrm{B} \\
\mathrm{A} \\
\mathrm{AB}\end{array}$ & $\begin{array}{l}\mathrm{BC} \\
\mathrm{B}\end{array}$ & $\begin{array}{l}\mathrm{B} \\
\mathrm{BC} \\
\mathrm{BD}\end{array}$ & $\begin{array}{l}\mathrm{C} \\
\mathrm{BC} \\
\mathrm{C}\end{array}$ & $\begin{array}{l}\mathrm{C} \\
\mathrm{C} \\
\mathrm{BC}\end{array}$ & $\begin{array}{l}\mathrm{ABC} \\
\mathrm{BC}\end{array}$ & $\begin{array}{l}\text { poly- } \\
\text { cyto. } 2 \mathrm{x}\end{array}$ \\
\hline $\begin{array}{l}\text { A. eriantha f. alba } \\
\text { A. fulvicoma var. fulvicoma } \\
\text { A. grandiflora }\end{array}$ & $\begin{array}{l}\mathrm{C} \\
\mathrm{BC} \\
\mathrm{BC}\end{array}$ & $\begin{array}{l}\mathrm{C} \\
\mathrm{BCD} \\
\mathrm{ABC} \\
\mathrm{C}\end{array}$ & $\begin{array}{l}\text { CD } \\
\text { CD } \\
\text { BC } \\
\text { DE }\end{array}$ & $\begin{array}{l}\mathrm{B} \\
\mathrm{BC}\end{array}$ & $\begin{array}{l}\mathrm{A} \\
\mathrm{AB} \\
\mathrm{A} \\
\mathrm{AB}\end{array}$ & $\begin{array}{l}\mathrm{AB} \\
\mathrm{A} \\
\mathrm{AB} \\
\mathrm{B}\end{array}$ & $\begin{array}{l}\mathrm{AB} \\
\mathrm{BCD} \\
\mathrm{AB} \\
\mathrm{BD}\end{array}$ & $\begin{array}{l}\mathrm{BC} \\
\mathrm{BC} \\
\mathrm{A} \\
\mathrm{BC}\end{array}$ & $\begin{array}{l}\mathrm{BC} \\
\mathrm{ABC} \\
\mathrm{C}\end{array}$ & $\begin{array}{l}\mathrm{AC} \\
\mathrm{ABC} \\
\mathrm{ABC}\end{array}$ & $\begin{array}{l}\text { cyto. } 2 \mathrm{x} \\
\text { poly- } \\
\text { poly- }\end{array}$ \\
\hline $\begin{array}{l}\text { A. guilinensis } \\
\text { A. hubeiensis } \\
\text { A. latifolia } \text { var. latifolia }\end{array}$ & $\begin{array}{l}\mathrm{BC} \\
\mathrm{ABCD}\end{array}$ & $\begin{array}{l}\mathrm{C} \\
\mathrm{B} \\
\mathrm{C}\end{array}$ & $\begin{array}{l}\mathrm{BD} \\
\mathrm{CD} \\
\mathrm{ABCD}\end{array}$ & $\begin{array}{l}\mathrm{CD} \\
\mathrm{AC} \\
\mathrm{A}\end{array}$ & $\begin{array}{l}\mathrm{A} \\
\mathrm{ABC} \\
\mathrm{A}\end{array}$ & $\begin{array}{l}\mathrm{B} \\
\mathrm{B} \\
\mathrm{B}\end{array}$ & $\begin{array}{l}\text { B } \\
\text { ABC }\end{array}$ & $\begin{array}{l}\mathrm{C} \\
\mathrm{BCD} \\
\mathrm{BC}\end{array}$ & $\begin{array}{l}\mathrm{BC} \\
\mathrm{C} \\
\mathrm{AB}\end{array}$ & $\begin{array}{l}\mathrm{BC} \\
\mathrm{ABC} \\
\mathrm{AC}\end{array}$ & $\begin{array}{l}\text { poly- } \\
\text { poly- }\end{array}$ \\
\hline $\begin{array}{l}\text { A. liangguangensis } \\
\text { A. lijiangensis } \\
\text { A. styracifolia } \\
\text { A. zhejiangensis }\end{array}$ & $\begin{array}{l}\mathrm{ABC} \\
\underset{\mathrm{B}}{\mathrm{AB}}\end{array}$ & $\begin{array}{l}\mathrm{C} \\
\mathrm{B} \\
\mathrm{BC} \\
\mathrm{ABC}\end{array}$ & $\begin{array}{l}\text { ACD } \\
\text { RABD } \\
\text { BC } \\
\text { CD }\end{array}$ & $\begin{array}{l}\text { ACD } \\
\text { ABC } \\
\text { BD } \\
\text { R3B }\end{array}$ & $\begin{array}{l}\mathrm{A} \\
\mathrm{AB} \\
\mathrm{A} \\
\mathrm{A}\end{array}$ & $\begin{array}{l}\mathrm{BC} \\
\mathrm{BC} \\
\mathrm{B} \\
\mathrm{C}\end{array}$ & $\begin{array}{l}\mathrm{A} \\
\mathrm{AC} \\
\mathrm{A} \\
\mathrm{AC}\end{array}$ & $\begin{array}{l}\mathrm{C} \\
\mathrm{C} \\
\mathrm{A} \\
\mathrm{BC}\end{array}$ & $\begin{array}{l}\mathrm{BC} \\
\mathrm{C} \\
\mathrm{C} \\
\mathrm{C}\end{array}$ & $\begin{array}{l}\mathrm{ABC} \\
\mathrm{BC} \\
\mathrm{AB} \\
\mathrm{ABC}\end{array}$ & $\begin{array}{l}\text { poly- } \\
\text { poly- }\end{array}$ \\
\hline
\end{tabular}

†Cyto., ploidy has been previously determined by cytogenetic study; poly-, polyploidy was revealed by allozymes only in this study.

‡Irregularly spaced position of bands designated as putative rare alleles starting at number 1 for the most anodal one. 
normally found in $A$. deliciosa and $A$. chinensis, two zones of enzyme activity, one anodal and one cathodal to the zone discussed above, were observed among taxa. As the genetic control of most enzymes is well enough known to allow genetic inference to be made from gel-banding patterns (Murphy et al., 1990), single- or double-banded phenotypes detected in these two zones allow us tentatively to designate two additional monomeric loci, $A c p-1$ and $A c p-3$, in Actinidia species. Most species possess either one or two ACP loci. However, it is interesting to note that A. melanandra Franch. (Sect. Leiocarpae Dunn) and A. callosa var. henryi Maxim. (Sect. Maculatae) possess all three ACP loci. Actinidia melanandra and $A$. callosa are the most widespread and presumptive progenitor species in the Sect. Leiocarpae and Sect. Maculatae, respectively (Cui, 1993).

\section{Peroxidase (PRX)}

Five zones of activity were observed for PRX (Fig. 1). In the JM family, segregation data for PRX-1, PRX-3 and PRX-4 confirmed the expected Mendelian ratios. This suggests a disomic mode of inheritance for these isozymes specified by three single genes (Prx-1, Prx-3 and Prx-4) (Table 1). However, the expected phenotypic ratios for tetrasomic inheritance were calculated and consistent with the assumption of a Mendelian mode of tetrasomic inheritance of five genes in the WE family (Table 2). The results showed the existence of two alleles at $P r x-1$ and $P r x-3$, and three alleles at $P r x-2, P r x-4$ and $P r x-5$. Of the 22 cultivars examined, 55 per cent of the cultivars were heterozygous at $P r x-1$ and $P r x-2$. A total of 18 per cent, 45 per cent and 68 per cent were heterozygous at $P r x-3, P r x-4$ and $P r x-5$, respectively (Table 3 ). Altogether, 46 per cent, 39 per cent, 63 per cent, 39 per cent and 52 per cent of the plants of 28 taxa were heterozygous at $P r x-1, P r x-2$, $P r x-3, P r x-4$ and $P r x-5$ respectively (Table 4$)$. Two additional zones of activity between PRX-3 and PRX-4 were observed among different species, which can be considered as species-specific rare isozymes. The zone cathodal to PRX-3 was detected in $A$. sabiae, $A$. latifolia, $A$. fasciculoides var. fasciculoides and $A$. deliciosa var. chlorocarpa. The zone anodal to PRX-4 was detected in $A$. callosa var. discolor, $A$. cylindrica var. reticulata, $A$. rubricaulis var. carica, $A$. hubeinensis, $A$. lijiangensis, $A$. fulvicoma, $A$. grandiflora, $A$. arguta and $A$. callosa var. henryi. The species of $A$. zhejiangensis, $A$. eriantha, $A$. eriantha f. alba, $A$. chrysantha and $A$. styracifolia exhibit both zones of isozyme activity. These rare isozymes appear to be observed mostly in species of the Sect. Maculatae.

\section{Phosphoglucoisomerase (PGI)}

PGI zymograms exhibited three zones of activity in Actinidia. PGI-1 was monomorphic in all Actinidia plants surveyed. PGI-3 was polymorphic, but poorly stained. This locus was subsequently excluded from further discussion. PGI-2 was polymorphic and well resolved: up to seven bands could be seen in individual plants carrying four alleles (tetra-allelic genotype). Triple-banded and five-banded phenotypes expressed by diallelic and triallelic genotypes could be reliably scored in the progenies of the crosses (Fig. 1). The observed ratios of progeny phenotypes were consistent with the expected phenotypic ratios for disomic inheritance in the JM (Table 1) and tetrasomic inheritance in the WE (Table 2) families. Five alleles, $a, b, c, d$ and $e$, could be assigned to the Pgi-2 locus. Of the 22 cultivars, 23 per cent, 27 per cent and 50 per cent were tri-, tetra- and diallelic heterozygotes, respectively. None of the cultivars and taxa examined was found to be homozygous for any single allele of Pgi-2 (Tables 3 and 4). These results are in agreement with the PGI phenotypes of triple-, five- and seven-banded patterns of $A$. deliciosa cultivars reported by Messina et al. (1991). In all, 55 per cent, 27 per cent and 18 per cent of the plants of the 28 taxa were di-, tri- and tetra-allelic heterozygotes (Table 4). A band anodal to allele $a$ was found only in $A$. callosa var. henryi and designated putatively as a rare allele $(\mathrm{R})$.

\section{Phosphoglucomutase (PGM)}

Two zones with PGM activity were observed in Actinidia species. PGM-1 was monomorphic in all plants of this study. PGM-2 was polymorphic with single-, double- and triple-banded patterns. Segregation data for the JM and WE families, in which the expected phenotypic ratios were tested for disomic and tetrasomic inheritance, respectively, confirmed the presence of three alleles, $b, c$ and $d$, at Pgm-2 (Tables 1 and 2). One regularly spaced band anodal to the position of the $b$ allele and two bands cathodal to the position of the $d$ allele were found in plants surveyed among 28 taxa and were designated putative alleles $a, e$ and $f$. Four additional bands were occasionally observed among taxa and were designated rare alleles R1-R4. Of the cultivars examined, 14 per cent, 32 per cent and 50 per cent were tetra-, di- and triallelic heterozygotes, respectively. Only 4 per cent of the cultivars were homo- 
zygous at the $b$ allele (Table 3). Among 28 taxa, 4 per cent of the plants were homozygous for the $a$ and $c$ alleles; 21 per cent were homozygous for the $b$ allele (Table 4). Alleles $f$ and $g$ appeared to be unique alleles in the Sect. Leiocarpae. Alleles R1-4 were limited to the Sect. Maculatae, with the exception of $\mathrm{R} 3$, in $A$. zhejiangensis and $\mathrm{R} 4$ in $A$. melanandra.

\section{Triosephosphate isomerase (TPI)}

One polymorphic TPI zone was observed in Actinidia species (Fig. 1). Regular segregation for tetrasomic inheritance was detected at the $a, b$ and $c$ alleles of this Tpi locus in the WE family (Table 2). Alleles $b$ and $c$ were also found to segregate normally in a 1:2:1 disomic mode of inheritance in the JM family (Table 1). A significantly high level of heterozygosity was observed among cultivars, as no homozygotes were found for any single allele. Allele $b$ is apparently predominant at the TPI locus. A total of 86 per cent of the cultivars were heterozygous, but had the $b$ allele in common (Table 3 ). Some 55 per cent of the cultivars were diallelic genotypes, 32 per cent and 9 per cent were tri- and tetra-allelic genotypes, respectively. Only 5 per cent of the plants of the 28 taxa were found to be homozygous for the $a$ allele, whereas 4 per cent were homozygous for the $b$ allele and 56 per cent and 29 per cent of the plants were tri- and diallelic heterozygotes, respectively. Tetra-allelic heterozygotes of Tpi were rare in natural Actinidia populations.

\section{Determining ploidy level}

Although kiwifruit ( $A$. deliciosa) has often been used as an example of the successful development of a worldwide fruit industry from a wild species in this century, definitive evidence is lacking as to whether $A$. deliciosa is an allo- or autohexaploid. Another commercialized species, $A$. chinensis, has both diploid and tetraploid races (Yan et al., 1994), but the origin of the tetraploid, $A$. chinensis, remains unclear. Cytogenetic studies determining the ploidy level have been hindered by the very small size and numerous number of chromosomes in Actinidia species (Ferguson, 1990). Allelic segregation analysis is the most definitive method of distinguishing autofrom alloploidy (Krebs \& Hancock, 1989). The allelic segregation observed at 10 isozyme loci presented above provides convincing evidence that $A$. deliciosa is an allohexaploid species, whereas the tetraploid, $A$. chinensis, is an autotetraploid that apparently originated from its diploid ancestor of the same species. It is interesting to note that a high percentage of selected cultivars are tetraploids. Ten of $12 \mathrm{~A}$. chinensis cultivars appear to be tetraploid, because triallelic or tetra-allelic phenotypes were found for at least one locus (Table 3 ). This is much higher than is usually found in natural populations (Yan et al., 1994). Further studies are needed to determine whether or not the polyploid nature is related to some desirable traits in $A$. chinensis. Polyploidy has been studied in about a dozen species of Actinidia (Table 4) (Xiong et al., 1985; McNeilage \& Considine, 1989). Allozyme analysis provides an inexpensive and efficient alternative way of detecting polyploidy when isozyme genetics become known in Actinidia. The results presented in Table 4 show that allozyme analysis for the detection of polyploidy was consistent with results obtained from cytogenetic studies. Ten additional species, which have not been studied for ploidy level, were revealed as polyploids by allozyme phenotypes in this study (Table 4). However, further confirmation from cytological studies is needed. Allelic segregation analysis has also been demonstrated effectively in distinguishing auto- from allopolyploidy in other plant species (Soltis \& Soltis, 1987; Krebs \& Hancock, 1989).

Ten isozyme loci verified in this study have provided useful markers for the further study of genetics and marker-aided selection in breeding programmes of commercial Actinidia species. Allozyme phenotyping should be a feasible technique for cultivar identification, because the polyploid nature of most Actinidia species precludes allozyme genotyping for cultivar identification. Significant isozymic variation was observed in plants of Actinidia, particularly in cultivars. All cultivars can be uniquely identified by any combination of three loci. The high percentage of di-, tri- or tetra-allelism found in allohexaploid $A$. deliciosa and autotetraploid $A$. chinensis indicated an effective maintenance of heterozygosity through polyploidy in Actinidia. As gene frequencies cannot be calculated from polyploids in natural populations even when using codominant allozymes, studies of phylogenetic relationships between species, although necessary, could be very difficult. Alternatively, allozyme phenotype frequencies might be used, although this can result in biased estimates of gene frequencies (for example, the tetraploid $\mathrm{BC}$ phenotype could have a $b b b c, b b c c$ or $b c c c$ genotype) and needs careful consideration.

\section{Acknowledgements}

This research was supported by the Chinese Academy of Sciences and the China National 
Commission of Education. The senior author is grateful to Dr D. L. Mulcahy for his generous help with the isoelectric focusing technique.

\section{References}

ANDERSON, C. M., CASTle, w. S. AND MOOR, G. A. 1991. Isozymic identification of zygotic seedlings in Swingle Citrumelo Citrus paradisi $\times$ Poncitrus trifoliata nursery and field populations $J$. Am. Soc. Hort. Sci., 116, $322-326$.

CROWHURST, R. N. AND GARDNER, R. C. 1991. A genomespecific repeat sequence from kiwifruit (Actinidia deliciosa var. deliciosa). Theor. Appl. Genet., 81, 71-78.

Cul, Z.-X. 1993. Actinidia in China. Shandong Scientific Press, Jinan, Shandong, China.

DEGANI, C., BEILES, A., EL-BATSRI, R., GOREN, M. AND GAZIT, s. 1995. Identifying lychee cultivars by isozyme analysis. J. Am. Soc. Hort. Sci., 120, 307-312.

FERGUSON, A. R. 1990. The genus Actinidia. In: Warrington, I. J. and Weston, G. C. (eds). Kiwifruit: Science and Management, pp. 15-35. Ray Richards Publisher, Auckland, New Zealand.

huANG, H., DANE, F. AND NORTON, J. D. 1994. Genetic analysis of 11 polymorphic isozyme loci in chestnut species and characterization of chestnut cultivars by multi-locus allozyme genotypes. J. Am. Soc. Hort. Sci., $119,840-849$.

KREBS, S. L. AND HANCOCK, J. F. 1989. Tetrasomic inheritance of isozyme markers in the highbush blueberry, Vaccinium corymbosum L. Heredity, 63, 11-18.

LIANG, C.-F. 1983. The distribution of Actinidia. Guihaia, 3, 229-248.

McNeIlage, M. A. AND Considine, J. A. 1989. Chromosome studies in some Actinidia taxa and implications for breeding. N. Z. J. Bot., 27, 71-81.

MANGANARIS, A. G., ALSTON, F. H., WEEDEN, N. F., ALDWINCKLE, H. S., GUSTAFSON, H. L. AND BROWN, S. K. 1994. Isozyme locus $P g m-1$ is tightly linked to a gene $\left(\mathrm{V}_{\mathrm{f}}\right)$ for scab resistance in apple. J. Am. Soc. Hort. Sci., 119, 1286-1288.

MESSINA, R., TESTOLIN, R. AND MORGANTE, M. 1991. Isozymes for cultivar identification in kiwifruit. HortScience, 26, 899-902.

MULCAHY, D. L., ROBINSON, R. W., IHARA, M., AND KESSELL, R. 1981. Gametophytic transcription for acid phosphatase in pollen of Cucurbita species hybrids. J. Hered., 72, 353-354

MURPHY, R. M., SITES, J. W., BUTH, D. G., JR AND HAUFER, C. H. 1990. Protein 1: Isozyme electrophoresis. In: Hillis, D. M. and Moritz, C. (eds) Molecular Systematics. Sinauer Associates, Sunderland, MA.

SOLTIS, D. E. AND soltis, P. S. 1987. Electrophoretic evidence for tetrasomic segregation in Tolmiea menziesii (Saxifragaceae). Heredity, 60, 375-382.

TANKSLEY, S. D. AND ORTON, T. J. 1983. Isozymes in Plant Genetics and Breeding. Elsevier, Amsterdam.

WANG, s., HUANG, R., WU, X. AND LING, K. 1994. Studies on Actinidia breeding by species hybridization. J. Fruit Sci., 11, 23-26.

WENDEL, J. F. AND WEEDEN, N. F. 1989. Visualization and interpretation of plant isozymes. In: Soltis, D. E. and Soltis, P. S. (eds) Isozymes in Plant Biology, pp. 9-33. Dioscorides Press, Portland, OR.

XIONG, Z.-T., HUANG, H. AND WU, X. 1985. Observations on the chromosome numbers of 4 species in Actinidia. $J$. Wuhan Bot. Res., 3, 219-224.

YAN, G., FERGUSON, A. R. AND McNeIlage, M. A. 1994. Ploidy races in Actinidia chinensis. Euphytica, 78, 175-183. 